\title{
Solving an inverse problem concerning the sheet metal blank geometry in an industrial application to minimize the processing time
}

\author{
Simon Mayr*§, Christian Zehetner ${ }^{\S}$, Christian Reisinger ${ }^{\S}$ and Wolfgang Kunze ${ }^{\dagger}$ \\ $\S$ University of Applied Sciences Upper Austria \\ Stelzhamerstraße 23, 4600 Wels, Austria \\ e-mail: Simon.Mayr2@fh-wels.at, web page : https://www.fh-ooe.at/ \\ $\uparrow$ Salvagnini Maschinenbau $\mathrm{GmbH}$ \\ Dr. Guido Salvagnini-Straße 1, 4482 Ennsdorf, Austria \\ e-mail: wolfgang.kunze@salvagninigroup.com, web page: https://www.salvagnini.at/
}

\begin{abstract}
This paper concerns the optimization of an industrial sheet metal forming process on an automatic panel bender from Salvagnini Maschinenbau $\mathrm{GmbH}$ [1] combined with a corner former from an external company. The considered process consists of four steps: (1) The sheet blank is cut out of the raw material. (2) The sheet is bent to $90^{\circ}$ on all four sides. (3) The area around the corners is formed into a full corner without welding. (4) Overlapping material at the corners must be cut off. The final product is a box with a precisely defined height and closed corners.

Objective of this work is to develop a simulation framework to find the optimal contour of the sheet blank, so that after bending (step 2) and corner forming (step 3) a perfect geometry is obtained without a final cutting step (4). For the automatic panel bender, a high reliable digital twin already exists[2]. The underlying FEM-model has been extended to simulate the bending process of all 4 sides. Secondly, a second FEM model has been created to represent the corner forming step. With these FEM-models, the process of step (2) and (3) can be simulated. Goal now is to find the optimum shape as an input of step (2) so that the output quality of step (3) does not require an additional step (4).

This inverse problem is solved iteratively: First, we assume a blank contour as initial data. After obtaining the results for bending and corner forming, the sheet blank is adapted. This cycle is repeated until the quality requirements for the corners are achieved. It turns out that with few iterations a suitable result for the industrial application is achieved. The modularized design of the developed simulation framework allows efficient adaptions to the specific requirements.
\end{abstract}

\section{REFERENCES}

[1] Salvagnini Maschinenbau GmbH, "Smart Panel Bender," 2021. https://www.salvagnini.at/produkte/biegen/p4lean/funktionsweise (accessed Feb. 01, 2021).

[2] C. Zehetner et al., "High-quality sheet metal production using a model-based adaptive approach," Procedia Comput. Sci., vol. 180, no. 2019, pp. 249-258, 2021. 